\title{
On generalized $Q$-systems
}

\section{Zoltán Bajnok, ${ }^{a}$ Etienne Granet, ${ }^{b, c}$ Jesper Lykke Jacobsen ${ }^{b, c, d}$ and Rafael I. Nepomechie ${ }^{e}$}

${ }^{a}$ Wigner Research Centre for Physics, Konkoly-Thege Miklós u. 29-33, 1121 Budapest, Hungary

${ }^{b}$ Institut de Physique Théorique, Paris Saclay, CEA, CNRS, 91191 Gif-sur-Yvette, France

${ }^{c}$ Laboratoire de Physique de l'Ecole Normale Supérieure, ENS, Université PSL, CNRS, Sorbonne Université, Université Paris-Diderot, Sorbonne Paris Cité, Paris, France

${ }^{d}$ Sorbonne Université, École Normale Supérieure, CNRS, Laboratoire de Physique (LPENS), 75005 Paris, France

e Physics Department, University of Miami, P.O. Box 248046, Coral Gables, FL 33124, U.S.A.

E-mail: bajnok.zoltan@wigner.hu, etienne.granet@physics.ox.ac.uk, jesper.jacobsen@ens.fr, nepomechie@miami.edu

ABStract: We formulate $Q$-systems for the closed XXZ, open XXX and open quantumgroup-invariant XXZ quantum spin chains. Polynomial solutions of these $Q$-systems can be found efficiently, which in turn lead directly to the admissible solutions of the corresponding Bethe ansatz equations.

KEYwords: Bethe Ansatz, Lattice Integrable Models

ARXiv EPrint: 1910.07805 


\section{Contents}

1 Introduction 1

2 Closed XXX $Q$-system 1

2.1 Review of the algebraic BA solution 1

2.2 Q-system 3

2.2.1 $Q_{a, s}$ in terms of $Q$ and $P \quad 4$

2.2.2 Construction of $P \quad 5$

$\begin{array}{lll}2.2 .3 & \text { An example } & 7\end{array}$

3 Closed XXZ $Q$-system $\quad 8$

3.1 Review of the algebraic BA solution 9

$\begin{array}{lll}3.2 & Q \text {-system and its solution } & 10\end{array}$

$\begin{array}{ll}\text { 3.2.1 An example } & 12\end{array}$

4 Open XXX $Q$-system 13

$\begin{array}{lll}4.1 & \text { Review of the algebraic BA solution } & 13\end{array}$

$\begin{array}{lll}4.2 & Q \text {-system } & 15\end{array}$

$\begin{array}{lll}\text { 4.2.1 } & Q_{a, s} \text { in terms of } Q \text { and } P & 16\end{array}$

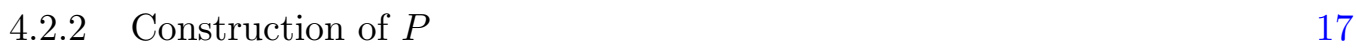

$\begin{array}{lll}4.2 .3 & \text { An example } & 18\end{array}$

5 Open quantum-group-invariant XXZ Q-system 18

$\begin{array}{lll}5.1 \text { Review of the algebraic BA solution } & 19\end{array}$

$\begin{array}{lll}5.2 & Q \text {-system } & 20\end{array}$

5.2.1 $Q_{a, s}$ in terms of $Q$ and $P \quad 21$

5.2.2 Construction of $P \quad 21$

$\begin{array}{lll}5.2 .3 & \text { An example } & 22\end{array}$

6 Conclusions $\quad 23$

A Exceptional solutions for open spin chains $\quad 23$

$\begin{array}{lll}\text { A.1 XXX } & 23\end{array}$

$\begin{array}{lll}\text { A.1.1 } & u_{1}=0 & 23\end{array}$

A.1.2 $u_{1}=\frac{i}{2} \quad 23$

$\begin{array}{lll}\text { A.2 XXZ } & 24\end{array}$ 


\section{Introduction}

An inconvenient truth about quantum integrable models - well-known to experts but seldom acknowledged - is that the corresponding Bethe ansatz (BA) equations (to which exact solutions of such models invariably reduce) are very difficult to solve. Various approaches to solving BA equations have been investigated, see e.g. [1, 2] and references therein. Significant further progress on this problem was recently achieved in [3], which formulated so-called $Q$-systems, whose polynomial solutions can be found efficiently; the zeros of the fundamental $Q$-function are the sought-after Bethe roots. The SU(2)-invariant $Q$-system was an essential ingredient in the recent computation of torus partition functions $[4,5]$, which exploited also techniques from algebraic geometry.

The $Q$-systems in [3] were restricted to rational BA equations for closed spin chains with periodic boundary conditions. The purpose of this paper is to generalize the $\mathrm{SU}(2)$ invariant $Q$-system [3] in two different directions: from rational to trigonometric, and from closed to open. These new $Q$-systems will be used to compute partition functions for trigonometric vertex models and for vertex models with boundaries [6, 7].

The outline of this paper is as follows. In section 2, we review the $Q$-system from [3] for the closed XXX spin chain with periodic boundary conditions. However, we provide an alternative derivation based on [8], which is convenient for deriving generalizations. In section 3 , we formulate a $Q$-system for the closed XXZ spin chain with periodic boundary conditions. We then turn to open spin chains. In section 4 , we formulate a $Q$-system for the SU(2)-invariant open XXX spin chain. A $Q$-system for the quantum-group-invariant open XXZ spin chain [9] is formulated in section 5. We conclude in section 6 with a brief summary and a list of some interesting open problems.

\section{Closed XXX $Q$-system}

In this section we review the $Q$-system [3] whose polynomial solutions provide the full spectrum of the closed XXX spin chain of length $N$ with periodic boundary conditions, whose Hamiltonian is given by

$$
\mathbb{H}=\sum_{k=1}^{N} \vec{\sigma}_{k} \cdot \vec{\sigma}_{k+1}, \quad \vec{\sigma}_{N+1} \equiv \vec{\sigma}_{1} .
$$

First the model is introduced and its solution by the algebraic BA method is recalled, together with the physicality conditions for the Bethe roots. We then describe the relevant $Q$-system and construct its solution explicitly. We show that polynomial solutions are in one-to-one correspondence with the physical solutions.

\subsection{Review of the algebraic BA solution}

The closed spin-1/2 XXX spin chain and its solution can be succinctly formulated with the help of an SU(2)-invariant solution of the Yang-Baxter equation given by the $4 \times 4$ $R$-matrix (see e.g. [10])

$$
\mathbb{R}(u)=\left(u-\frac{i}{2}\right) \mathbb{I}+i \mathbb{P},
$$


where $\mathbb{P}$ is the permutation matrix, $\mathbb{I}$ is the identity matrix, and $u$ is the spectral parameter. For $N$ sites with periodic boundary conditions, one can introduce the monodromy matrix $\mathbb{M}$ and the transfer matrix $\mathbb{T}$ as

$$
\mathbb{T}(u)=\operatorname{tr}_{0}\left(\mathbb{M}_{0}(u)\right), \quad \mathbb{M}_{0}(u)=\mathbb{R}_{01}(u) \mathbb{R}_{02}(u) \ldots \mathbb{R}_{0 N}(u)
$$

An auxiliary space denoted by index 0 has been introduced, and lower indices help indicate the spaces in which operators act. The transfer matrix is obtained by tracing over the auxiliary space, and thus acts on the quantum space, which is the $N$-fold tensor product of $\mathbb{C}^{2}$ accommodating all possible states of spin-up and spin-down. As a consequence of the Yang-Baxter equation, the transfer matrix forms a one-parameter family of commuting operators

$$
[\mathbb{T}(u), \mathbb{T}(v)]=0,
$$

and generates conserved charges in involution, including the Hamiltonian of the system (2.1).

We are interested in the eigenvectors and eigenvalues of the transfer matrix. The former can be generated from the all spin-up reference state

$$
|0\rangle=\left(\begin{array}{l}
1 \\
0
\end{array}\right)^{\otimes N}
$$

by acting with a matrix element of the monodromy matrix as

$$
\mathbb{B}\left(u_{1}\right) \ldots \mathbb{B}\left(u_{M}\right)|0\rangle \equiv\left|u_{1}, \ldots, u_{M}\right\rangle, \quad \mathbb{M}_{0}(u)=\left(\begin{array}{c}
\mathbb{A}(u) \mathbb{B}(u) \\
\mathbb{C}(u) \mathbb{D}(u)
\end{array}\right)
$$

The eigenvalues $T(u)$ of the transfer matrix

$$
\mathbb{T}(u)\left|u_{1}, \ldots, u_{M}\right\rangle=T(u)\left|u_{1}, \ldots, u_{M}\right\rangle
$$

satisfy the $T Q$-relation

$$
T(u) Q(u)=\left(u+\frac{i}{2}\right)^{N} Q(u-i)+\left(u-\frac{i}{2}\right)^{N} Q(u+i),
$$

where $Q$ encodes the Bethe roots $\left\{u_{i}\right\}$ :

$$
Q(u)=\prod_{j=1}^{M}\left(u-u_{j}\right)
$$

As follows from the definition of the transfer matrix and its commutativity property (2.4), $T(u)$ is a polynomial in $u$, and is thus regular at $u_{j}$. The $T Q$-relation $(2.8)$ then leads to the BA equations for the roots:

$$
\left(\frac{u_{j}+\frac{i}{2}}{u_{j}-\frac{i}{2}}\right)^{N}=-\prod_{k=1}^{M} \frac{u_{j}-u_{k}+i}{u_{j}-u_{k}-i}, \quad j=1, \ldots, M .
$$

For roots with multiplicities, we have further equations $[11,12]$. Since repeated roots do not seem to appear in this model (see e.g. [2]), we assume that roots never coincide. 
We call a solution of the BA equations physical if the corresponding Bethe vector (2.6) is an eigenvector of the transfer matrix. Unfortunately, not all solutions of the BA equations are physical. Solutions that contain the roots $\pm \frac{i}{2}$ can be unphysical, i.e. they might solve the BA equations, but there is no related eigenvector of the transfer matrix. We define a solution $\left\{u_{1}, \ldots, u_{M}\right\}$ of the BA equations to be admissible, if all roots are finite and pairwise distinct; and, if they are of the form $\left\{\frac{i}{2},-\frac{i}{2}, u_{1}, \ldots, u_{M-2}\right\}$ (which we call a singular solution), then the further constraint

$$
\prod_{j=1}^{M-2} \frac{\left(u_{j}+\frac{i}{2}\right)}{\left(u_{j}-\frac{i}{2}\right)} \frac{\left(u_{j}+\frac{3 i}{2}\right)}{\left(u_{j}-\frac{3 i}{2}\right)}=(-1)^{N}
$$

is satisfied. It was shown in [13] that admissibility implies physicality, and the converse follows from Lemmata 2 and 4 of [8]. Hence, admissibility and physicality are equivalent. The number $\mathcal{N}(N, M)$ of admissible solutions of the BA equations with $M \leq N / 2$ has been conjectured to be given by (see e.g. [2])

$$
\mathcal{N}(N, M)=\left(\begin{array}{l}
N \\
M
\end{array}\right)-\left(\begin{array}{c}
N \\
M-1
\end{array}\right)
$$

Alternatively, it was observed in [3] that the polynomial solutions of a $Q$-system on an appropriately chosen diagram can be computed efficiently, and correctly account for the physical solutions. In the remainder of this section, we provide an alternative derivation of these results based on [8], which we will subsequently use to generalize this $Q$-system.

\section{$2.2 \quad Q$-system}

For given values of $N$ and $M$, the $Q$-functions $Q_{a, s}$ are defined on a Young diagram with the indices referring to the vertex $(a, s)$, where the $a$-axis is vertical and the $s$-axis is horizontal, see figure 1 . These $Q$-functions satisfy the $Q Q$-equations, which are formulated around a face as $^{1}$

$$
Q_{a+1, s}(u) Q_{a, s+1}(u) \propto Q_{a+1, s+1}^{+}(u) Q_{a, s}^{-}(u)-Q_{a+1, s+1}^{-}(u) Q_{a, s}^{+}(u),
$$

where $f^{ \pm}(u)=f\left(u \pm \frac{i}{2}\right)$. The relevant diagram for the closed XXX spin chain with the boundary conditions, $Q_{2, s}=1, Q_{1, s \geq M}=1$, is displayed in figure 1 . The initial condition

$$
Q_{0,0}(u)=u^{N}, \quad Q_{1,0}(u)=Q(u)=\prod_{j=1}^{M}\left(u-u_{j}\right),
$$

leads to a unique solution of the $Q$-system. The degree of the polynomial $Q_{a, s}(u)$ is given by the number of boxes in the Young diagram to the right and top of the vertex $(a, s)$. Let us see how we can proceed column-by-column and express all $Q$-functions in terms of $Q_{0,0}$ and $Q_{1,0}$.

The $Q Q$-equation for $(a, s)=(1,0)$ can be solved easily

$$
Q_{1,1}(u)=Q_{1,0}^{+}(u)-Q_{1,0}^{-}(u) \equiv Q_{1,0}^{\prime}(u)=Q^{\prime}(u),
$$

\footnotetext{
${ }^{1} \mathrm{~A} Q$-function is defined up to a multiplicative constant. For definiteness, we generally treat $\propto$ as equality, or (as in (2.15)) with an extra minus sign.
} 


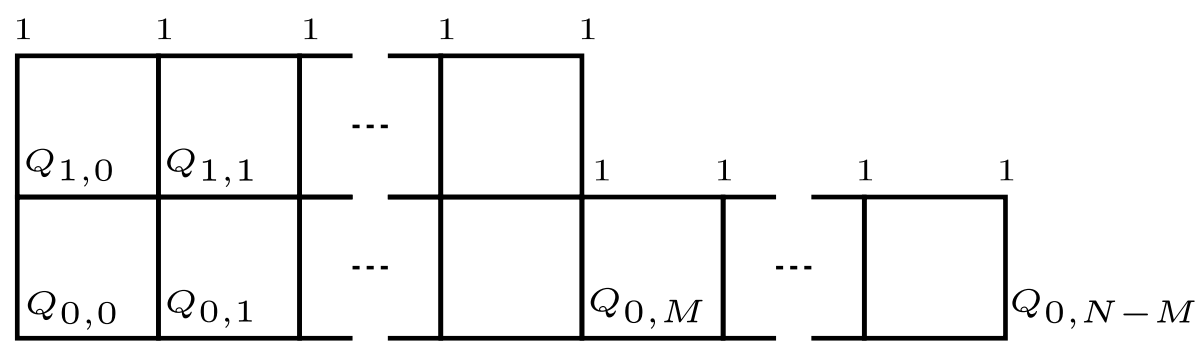

Figure 1. Non-trivial $Q$-functions for the closed XXX spin chain.

where we have introduced the discrete derivative defined by

$$
f^{\prime}(u)=f^{+}(u)-f^{-}(u)
$$

The function $Q_{1,1}$ is automatically a polynomial of degree $M-1$. The equation for $(a, s)=(0,0)$ gives

$$
Q_{0,1} Q_{1,0}=Q_{0,0}^{-} Q_{1,1}^{+}-Q_{0,0}^{+} Q_{1,1}^{-}
$$

Making use of (2.15) and (2.17), it follows that

$$
Q_{0,1} Q=Q_{0,0}^{-} Q^{++}+Q_{0,0}^{+} Q^{--}-Q\left(Q_{0,0}^{-}+Q_{0,0}^{+}\right)
$$

or

$$
\left(Q_{0,1}+Q_{0,0}^{-}+Q_{0,0}^{+}\right) Q=Q_{0,0}^{-} Q^{++}+Q_{0,0}^{+} Q^{--} .
$$

Recognizing the r.h.s. of the above equation as the r.h.s. of the $T Q$-relation (2.8), one obtains

$$
T=Q_{0,1}+Q_{0,0}^{-}+Q_{0,0}^{+} .
$$

Polynomiality of $Q_{0,1}$ is equivalent to the polynomiality of $T$, which leads to the BA equations (2.10).

\subsection{1 $Q_{a, s}$ in terms of $Q$ and $P$}

We now show that the polynomiality of the remaining $Q$-functions is equivalent to the admissibility of $\left\{u_{1}, \ldots, u_{M}\right\}$. To this end, we define a function $P(u)$, such that

$$
Q_{0,0}=P^{+} Q^{-}-P^{-} Q^{+} .
$$

Using this parametrization for $Q_{0,0}$, one can easily show that

$$
Q_{0,1} \propto P^{\prime+} Q^{\prime-}-P^{\prime-} Q^{\prime+},
$$

where prime denotes discrete derivative (2.16). Repeating the calculations starting from $(a, s)=(1,1)$ and $(a, s)=(0,1)$, we arrive at

$$
Q_{1,2}=Q^{\prime \prime}, \quad Q_{0,2} \propto P^{\prime \prime+} Q^{\prime \prime-}-P^{\prime \prime-} Q^{\prime \prime+} .
$$


This can be iterated further

$$
Q_{1, n}=Q^{(n)}, \quad Q_{0, n} \propto P^{(n)+} Q^{(n)-}-P^{(n)-} Q^{(n)+},
$$

where the superscript $(n)$ denotes the $n^{\text {th }}$ discrete derivative. In short, all $Q$-functions can be expressed in terms of $P$ and $Q$. Clearly, if $P$ is a polynomial, then all $Q$-functions are polynomial. In the following we show that the polynomiality of $P$ is in fact equivalent to the polynomiality of $Q_{0,2}$. We also derive that polynomiality of $P$ is equivalent to the admissibility of the roots $\left\{u_{1}, \ldots, u_{M}\right\}$.

\subsubsection{Construction of $P$}

We construct $P$ as in [8] by generalizing the approach in [14] (which implicitly assumes that all Bethe roots are regular) to the case of a singular solution. String configurations have roots that differ by $i: u_{i_{1}}-u_{i_{2}}=i$, and it is well known (see e.g. [8]) that the only exact string solution consists of one pair of singular roots $u_{1}=\frac{i}{2}$ and $u_{2}=-\frac{i}{2}$. In the presence of such singular roots, the $Q$-function takes the following form

$$
Q(u)=u^{+} u^{-} \bar{Q}(u), \quad \bar{Q}(u)=\prod_{u_{j} \neq \pm \frac{i}{2}}\left(u-u_{j}\right) .
$$

We start by dividing $(2.21)$ by $Q^{+} Q^{-}$. We need to write

$$
R(u)=\frac{u^{N}}{Q^{+} Q^{-}}=\frac{u^{N-2}}{u^{++} u^{--} \bar{Q}^{+} \bar{Q}^{-}}
$$

in the form

$$
R=\frac{P^{+}}{Q^{+}}-\frac{P^{-}}{Q^{-}}=\left(\frac{P}{Q}\right)^{\prime},
$$

i.e. we need to "integrate" $R$ in the discrete sense. To this end, we perform a partial fraction decomposition of $(2.26)$

$$
R=\pi+\frac{q_{+}}{\bar{Q}^{+}}+\frac{q_{-}}{\bar{Q}^{-}}+\frac{a_{+}}{u^{++}}+\frac{a_{-}}{u^{--}},
$$

where $\pi$ is a polynomial of order $N-2 M$, the polynomials $q_{ \pm}$have degree less than $\bar{Q}$, while $a_{ \pm}$are constants. Using the relation

$$
R^{+}+R^{-}=\frac{T}{Q^{++} Q^{--}}=\frac{T}{u^{+} u^{-} u^{+++} u^{---} \bar{Q}^{++} \bar{Q}^{--}}
$$

which follows from the $T Q$-relation (2.8), one can investigate all the singularities explicitly. In particular, the r.h.s. of (2.29) has no singularities at the zeros of $\bar{Q}$, implying

$$
q_{+}=q^{+}, \quad q_{-}=-q^{-},
$$

for some polynomial $q(u)$. The coefficients $a_{ \pm}$can be determined from the residues of (2.29) at $u=\mp \frac{i}{2}$ :

$$
a_{ \pm}=\mp \frac{T\left(\mp \frac{i}{2}\right)}{2 i \bar{Q}\left( \pm \frac{i}{2}\right) \bar{Q}\left(\mp \frac{3 i}{2}\right)} .
$$


The polynomial $\pi$ can always be written as

$$
\pi=\rho^{\prime}=\rho^{+}-\rho^{-},
$$

where $\rho$ is a polynomial. Clearly, $\rho$ is defined up to a constant. Changing this constant by $a$ modifies $P$ as $P+a Q$. This additional term, however, disappears from $R$ and $Q_{0,0}$, thus is irrelevant for us.

In the absence of singular roots, we have $\bar{Q}=Q$ and $a_{ \pm}=0$; hence, the polynomial $P=\rho Q+q$ satisfies (2.27), which implies the required eq. (2.21), see also [14]. In the presence of singular roots, the "integration" of $R$ in $(2.27)$ requires to "integrate" $u^{-1}$, appearing in (2.28). To this end, we define the function $p(u)$ by

$$
p^{\prime}(u)=\frac{1}{u}, \quad p(u)=-i \psi\left(-i u+\frac{1}{2}\right)
$$

where $\psi(u)$ is the digamma function

$$
\psi(u)=-\gamma+\sum_{n=0}^{\infty}\left(\frac{1}{n+1}-\frac{1}{n+u}\right) .
$$

In view of the fact

$$
\begin{aligned}
\frac{a_{+}}{u^{++}}+\frac{a_{-}}{u^{--}} & =\left(a_{+} p^{++}+a_{-} p^{--}\right)^{\prime}=\left[\frac{1}{2}\left(a_{+}-a_{-}\right)\left(p^{++}-p^{--}\right)+\frac{1}{2}\left(a_{+}+a_{-}\right)\left(p^{++}+p^{--}\right)\right]^{\prime} \\
& =\left[\frac{1}{2}\left(a_{+}-a_{-}\right)\left(\frac{1}{u^{+}}+\frac{1}{u^{-}}\right)+\frac{1}{2}\left(a_{+}+a_{-}\right)\left(p^{++}+p^{--}\right)\right]^{\prime}
\end{aligned}
$$

we see that the function $P$ satisfying (2.27) takes the form

$$
P=\rho Q+u^{+} u^{-} q+\left(a_{+}-a_{-}\right) u \bar{Q}+\frac{1}{2}\left(a_{+}+a_{-}\right)\left(p^{++}+p^{--}\right) Q .
$$

It is a polynomial if and only if $a_{+}=-a_{-}$, i.e. when

$$
(-1)^{M} \frac{\bar{Q}\left(+\frac{i}{2}\right) \bar{Q}\left(+\frac{3 i}{2}\right)}{\bar{Q}\left(-\frac{i}{2}\right) \bar{Q}\left(-\frac{3 i}{2}\right)}=1
$$

is satisfied. Here we used the $T Q$-relation (2.8) to eliminate $T\left( \pm \frac{i}{2}\right)$ in (2.31). Clearly this is the admissibility condition for singular solutions (2.11). Thus, we have just proven that polynomiality of $P$ is equivalent to the admissibility of the roots.

Even if $P$ is not a polynomial, the relation (2.27) implies (2.21), which leads to

$$
T=P^{++} Q^{--}-P^{--} Q^{++} .
$$

This implies that $P$ also satisfies the $T Q$-relation

$$
T P=\left(u^{+}\right)^{N} P^{--}+\left(u^{-}\right)^{N} P^{++} .
$$

Thus $P$ and $Q$ are the two independent solutions of this second order difference equation, and $(2.21)$ is the corresponding Wronskian relation. It has been known (see e.g. $[15,16]$ ) 
that the two independent solutions of the $T Q$-relation are both polynomial iff the Bethe state (2.6) is an eigenstate of the transfer matrix.

Finally, let us investigate the polynomiality of

$$
Q_{0,2}=Q_{0,1}^{+}+Q_{0,1}^{-}+P^{\prime++} Q^{\prime--}-P^{\prime--} Q^{\prime++} .
$$

Since $Q_{0,1}$ is a polynomial, ${ }^{2}$ we investigate the regularity of the remaining part at $u=0$. Since $Q\left( \pm \frac{i}{2}\right)=0$ we can see that

$$
Q^{\prime--}(0)=-Q\left(-\frac{3 i}{2}\right), \quad Q^{\prime++}(0)=Q\left(\frac{3 i}{2}\right)
$$

which are not zero. We now focus on the pole contributions at $u=0$. They can only come from the terms proportional to $p(u)$, which have poles at $u=-i\left(n+\frac{1}{2}\right)$ for any integer $n \geq 0$, with residues -1 . Thus the singular parts can arise as

$$
\begin{aligned}
P^{\prime++}(\epsilon) & =P\left(\frac{3 i}{2}+\epsilon\right)+\cdots=\frac{1}{2}\left(a_{+}+a_{-}\right) Q\left(\frac{3 i}{2}\right)\left(p^{+++++}(\epsilon)+p^{+}(\epsilon)\right)=0+\ldots \\
P^{\prime--}(\epsilon) & =-P\left(-\frac{3 i}{2}+\epsilon\right)+\cdots=-\frac{1}{2}\left(a_{+}+a_{-}\right) Q\left(-\frac{3 i}{2}\right)\left(p^{-----}(\epsilon)+p^{-}(\epsilon)\right) \\
& =\frac{1}{\epsilon}\left(a_{+}+a_{-}\right) Q\left(-\frac{3 i}{2}\right)+\ldots
\end{aligned}
$$

where we have omitted regular terms in $\epsilon$. The singular part of $Q_{0,2}(\epsilon)$ is then $-\frac{1}{\epsilon}\left(a_{+}+\right.$ $\left.a_{-}\right) Q\left(\frac{3 i}{2}\right) Q\left(-\frac{3 i}{2}\right)$ whose vanishing implies $a_{+}=-a_{-}$, i.e. the polynomiality of $P$.

We can thus conclude that the following four properties are equivalent:

(i) $P$ is a polynomial

(ii) all $Q_{a, s}$-functions are polynomial

(iii) the roots $\left\{u_{1}, \ldots, u_{M}\right\}$ are admissible solutions of the BA equations

(iv) the Bethe vector is an eigenvector of the transfer matrix

\subsubsection{An example}

We conclude this subsection with an elementary explicit example of how to use the Qsystem (2.13)-(2.14) to obtain all the Bethe roots for modest values of $N \equiv L$ and $M$ using a simple-minded implementation in Mathematica. (A more general and sophisticated code is provided in [3].) We begin by defining the functions $Q_{0,0}(u)$ and $Q_{1,0}(u)$

$\mathrm{Q}\left[0,0, \mathrm{u}_{-}\right]:=\mathrm{u}^{\wedge} \mathrm{L}$;

$Q\left[1,0, u_{-}\right]:=\operatorname{Sum}\left[c[k] u^{\wedge} k,\{k, 0, M-1\}\right]+u^{\wedge} M$;

\footnotetext{
${ }^{2}$ Recall from (2.20) that polynomiality of $Q_{0,1}$ is equivalent to polynomiality of $T$; and the latter is evident from

$$
T=\left(u^{+}\right)^{N-1} u^{---} \frac{\bar{Q}^{--}}{\bar{Q}}+\left(u^{-}\right)^{N-1} u^{+++} \frac{\bar{Q}^{++}}{\bar{Q}},
$$

which follows from the $T Q$-relation (2.8) and (2.25), and which has vanishing residues at the zeros of $\bar{Q}$ by virtue of the BA equations.
} 
where the coefficients $c_{k}$ are to be determined. We also define the functions $Q_{1, n}(u)$ and $Q_{0, n}(u)$ by $^{3}$

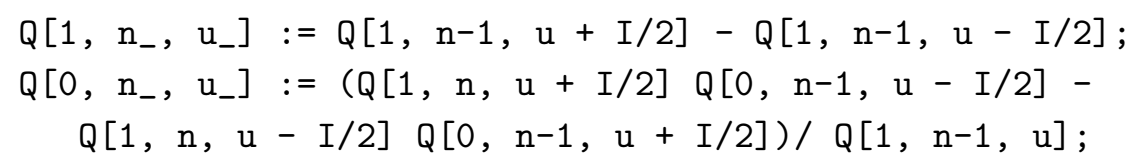

which follow from (2.13) with $a=1$ (setting $Q_{2, s}=1$ ) and $a=0$, respectively. The functions $Q_{1, n}(u)$ are evidently polynomials in $u$; the key point is that the functions $Q_{0, n}(u)$ must also be polynomials in $u$. In order to ensure the latter requirement (the so-called zeroremainder conditions), we first use the built-in symbol PolynomialRemainder to define the polynomials $y_{n}(u)$

$\mathrm{y}\left[\mathrm{n}_{-}, \mathrm{u}_{-}\right]:=$PolynomialRemainder [Numerator [Together $\left.[\mathrm{Q}[0, \mathrm{n}, \mathrm{u}]]\right]$,

Denominator [Together $[\mathrm{Q}[0, \mathrm{n}, \mathrm{u}]]], \mathrm{u}]$;

We then solve for the coefficients $c_{k}$ that make $\left\{y_{1}(u), \ldots, y_{M}(u)\right\}$ vanish for all values of $u,{ }^{4}$

sol = Solve $[$ Table $[$ CoefficientList $[y[n, u], u]==0,\{n, 1, M\}]$, Table $[\mathrm{c}[\mathrm{k}],\{\mathrm{k}, 0, \mathrm{M}-1\}]]$

Let us consider as an example the case $L=6, M=2$. The above code generates 9 solutions, in agreement with (2.12). For each of these solutions, one can obtain the corresponding Bethe roots by solving for the zeros of $Q_{1,0}(u)$. For example, for the first solution

Solve $[(Q[1,0, u] / . \operatorname{sol}[[1]])==0, u] / /$ Flatten

we obtain the Bethe roots $\pm i / 2$.

\section{Closed XXZ $Q$-system}

In this section we present a generalization of the $Q$-system for the closed XXZ spin chain of length $N$ with periodic boundary conditions, whose Hamiltonian is given by

$$
\mathbb{H}=\sum_{k=1}^{N}\left[\sigma_{k}^{x} \sigma_{k+1}^{x}+\sigma_{k}^{y} \sigma_{k+1}^{y}+\frac{1}{2}\left(q+q^{-1}\right) \sigma_{k}^{z} \sigma_{k+1}^{z}\right], \quad \vec{\sigma}_{N+1} \equiv \vec{\sigma}_{1} .
$$

We show that there is a notion of polynomial solutions of the $Q Q$-equations, which determine the spectrum of the closed XXZ model.

\footnotetext{
${ }^{3}$ The equation for $Q_{1, n}$ is valid only for $1 \leq n \leq M$. For $n=M$, we find $Q_{1, M} \propto 1$.

${ }^{4}$ For some examples, the coefficients $c_{k}$ can be determined using fewer than $M$ equations.
} 


\subsection{Review of the algebraic BA solution}

The XXZ spin chain is related to the trigonometric generalization of the rational $R$ matrix $(2.2):^{5}$

$$
\mathbb{R}(u)=\left(\begin{array}{cccc}
\sinh \left(u+\frac{\eta}{2}\right) & 0 & 0 & 0 \\
0 & \sinh \left(u-\frac{\eta}{2}\right) & \sinh (\eta) & 0 \\
0 & \sinh (\eta) & \sinh \left(u-\frac{\eta}{2}\right) & 0 \\
0 & 0 & 0 & \sinh \left(u+\frac{\eta}{2}\right)
\end{array}\right) .
$$

The monodromy and transfer matrices can be introduced by the analogous formulae to the XXX case (2.3). The off-diagonal elements of the monodromy matrix (2.6) can be used as creation and annihilation operators. The $\mathbb{B}$ operators, by acting on the all spin-up reference state $(2.5)$, create eigenstates of the transfer matrix. The eigenvalue $T(u)$ of the transfer matrix $\mathbb{T}(u)$ satisfies the $T Q$-relation

$$
T(u) Q(u)=\sinh ^{N}\left(u+\frac{\eta}{2}\right) Q(u-\eta)+\sinh ^{N}\left(u-\frac{\eta}{2}\right) Q(u+\eta),
$$

where now

$$
Q(u)=\prod_{j=1}^{M} \sinh \left(u-u_{j}\right) .
$$

Alternatively, we can switch to the variable $t=e^{u}$. By construction, $T(u)$ is a polynomial of $t$ and $t^{-1}$, regular at $t_{j}=e^{u_{j}}$, which implies the BA equations

$$
\left(\frac{\sinh \left(u_{k}+\frac{\eta}{2}\right)}{\sinh \left(u_{k}-\frac{\eta}{2}\right)}\right)^{N}=-\prod_{j=1}^{M} \frac{\sinh \left(u_{k}-u_{j}+\eta\right)}{\sinh \left(u_{k}-u_{j}-\eta\right)}, \quad k=1, \ldots, M
$$

Singular BA solutions appear also for the XXZ spin chain, and the admissibility of the solution $\left\{-\frac{\eta}{2}, \frac{\eta}{2}, u_{1}, \ldots, u_{M-2}\right\}$ with pairwise distinct and finite roots can be formulated similarly to the XXX case as [17]

$$
\frac{\bar{Q}\left(+\frac{\eta}{2}\right) \bar{Q}\left(+\frac{3 \eta}{2}\right)}{\bar{Q}\left(-\frac{\eta}{2}\right) \bar{Q}\left(-\frac{3 \eta}{2}\right)}=(-1)^{N},
$$

where $\bar{Q}(u)=\prod_{j=1}^{M-2} \sinh \left(u-u_{j}\right)$. We work for generic $\eta$, i.e. when $q=e^{\eta}$ is not a root of unity. We conjecture that the number $\mathcal{N}(N, M)$ of admissible solutions of the BA equations with $M \leq N / 2$ is given by

$$
\mathcal{N}(N, M)=\left(\begin{array}{l}
N \\
M
\end{array}\right)
$$

In the following we introduce a $Q$-system whose polynomial (in $t$ and $t^{-1}$ ) solutions account for the physical solutions.

\footnotetext{
${ }^{5}$ The XXX limit can be recovered by the rescalings $u \rightarrow \epsilon u, \eta \rightarrow i \epsilon$ and by then taking the $\epsilon \rightarrow 0$ limit.
} 


\section{2 $Q$-system and its solution}

For the closed XXZ case, as in the XXX case, $Q_{2, s}=1$ and the nontrivial $Q$-functions are $Q_{1, s}$ and $Q_{0, s}$; however, there is no condition on $Q_{1, s \geq M}$. We now regard the $Q$-functions as functions of the argument $t=e^{u}$. Moreover, shifts now denote $f^{ \pm}(t)=f\left(t q^{ \pm \frac{1}{2}}\right)$, and $Q Q$-equations are formulated around each face as

$$
Q_{a+1, s}(t) Q_{a, s+1}(t) \propto Q_{a+1, s+1}^{+}(t) Q_{a, s}^{-}(t)-Q_{a+1, s+1}^{-}(t) Q_{a, s}^{+}(t) .
$$

The initial conditions are

$$
Q_{0,0}(t)=\left(t-t^{-1}\right)^{N}, \quad Q_{1,0}(t)=Q(t)=\prod_{j=1}^{M}\left(t t_{j}^{-1}-t^{-1} t_{j}\right) .
$$

Both of these $Q$-functions are polynomial in the variables $t$ and $t^{-1}$. As in the XXX case, we introduce the analogue of $P$, and then proceed to express all $Q$-functions in terms of $P$ and $Q$.

The $Q Q$-equation for $(a, s)=(1,0)$ leads again to the discrete derivative of $Q$ :

$$
Q_{1,1}(t)=Q_{1,0}^{+}(t)-Q_{1,0}^{-}(t) \equiv Q_{1,0}^{\prime}(t)=Q^{\prime}(t) .
$$

However, contrary to the XXX case, the order of $Q_{1,1}$ is the same as that of $Q .{ }^{6}$ Since the $Q Q$-equations for the XXZ case (3.8) are the same as for the XXX case (2.13), the solutions are the same, too. In particular, formulas such as (2.18)-(2.20) are exactly the same, and polynomiality of $Q_{0,1}$ is equivalent to the polynomiality of $T$, which gives the BA equations (3.5). In proceeding as before, we search for a function $P$ that satisfies

$$
Q_{0,0}=P^{+} Q^{-}-P^{-} Q^{+},
$$

that is,

$$
\left(t-t^{-1}\right)^{N}=P\left(t q^{\frac{1}{2}}\right) Q\left(t q^{-\frac{1}{2}}\right)-P\left(t q^{-\frac{1}{2}}\right) Q\left(t q^{\frac{1}{2}}\right) .
$$

With this $P$ and $Q$, all $Q$-functions can be written as in the XXX case (2.24)

$$
Q_{1, n}=Q^{(n)}, \quad Q_{0, n} \propto P^{(n)+} Q^{(n)-}-P^{(n)-} Q^{(n)+},
$$

except that the superscript $(n)$ denotes the $n^{\text {th }}$ discrete derivative obtained from multiplicative shifts in $t$, with $f^{ \pm}(t)=f\left(t q^{ \pm \frac{1}{2}}\right)$ and $f^{\prime}(t)=f^{+}(t)-f^{-}(t)$.

The construction of the function $P$, once written in terms of the shifts, literally repeats the steps in the XXX case. One first shows that the only singular solutions are $t=q^{ \pm \frac{1}{2}}$. One then separates the singular solutions as $Q(t)=\left(t-t^{-1}\right)^{+}\left(t-t^{-1}\right)^{-} \bar{Q}(t)$, and performs a partial fraction decomposition of $R(t)$ as

$$
R(t)=\frac{\left(t-t^{-1}\right)^{N}}{Q^{+} Q^{-}}=\pi+\frac{r_{+}}{\bar{Q}^{+}}+\frac{r_{-}}{\bar{Q}^{-}}+\frac{a_{+}}{\left(t-t^{-1}\right)^{++}}+\frac{a_{-}}{\left(t-t^{-1}\right)^{--}},
$$

\footnotetext{
${ }^{6}$ In fact, $Q_{1, n}$ has order $M$, and $Q_{0, n}$ has order $N$.
} 
where $\pi(t)$ is polynomial. From the singularity structure, one can obtain $r_{+}=r^{+}$and $r_{-}=-r^{-}$for some polynomial function $r(t)$, together with

$$
a_{ \pm}= \pm \frac{2^{N} T\left(q^{\mp \frac{1}{2}}\right)}{\left(q-q^{-1}\right)^{2}\left(q^{2}-q^{-2}\right)} \frac{1}{\bar{Q}\left(q^{ \pm \frac{1}{2}}\right) \bar{Q}\left(q^{\mp \frac{3}{2}}\right)}
$$

The important new step now is the discrete integration of $\left(t-t^{-1}\right)^{-1}=\frac{1}{2}\left(\frac{1}{t-1}+\frac{1}{t+1}\right)$. To this end, we define the function $p_{q}(t)$ by

$$
p_{q}^{\prime}(t)=\frac{1}{t-t^{-1}}, \quad p_{q}(t)=\frac{1}{2 \log q}\left\{\psi_{q^{-1}}\left(\frac{\log t}{\log q}+\frac{1}{2}\right)-\psi_{q^{-1}}\left(\frac{\log (-t)}{\log q}+\frac{1}{2}\right)\right\}
$$

where $\psi_{q}(x)$ denotes the $q$-deformed digamma function $[18,19]$, which satisfies

$$
\psi_{q}(x+1)-\psi_{q}(x)=\frac{\log q}{1-q^{-x}} .
$$

Another step of the XXX case that requires special care when generalizing to the XXZ case, which was already addressed in [14], is the deformed discrete integration of $\pi$ into $\rho$ such that

$$
\rho^{\prime}=\pi
$$

The non-constant terms can be integrated using $\left(\frac{t^{n}}{q^{n / 2}-q^{-n / 2}}\right)^{\prime}=t^{n}$ for $n \neq 0$. However, a constant term cannot be integrated into a polynomial, and requires instead a function $c_{q}(t)$ such that $c_{q}^{\prime}(t)=1$. We have

$$
c_{q}(t)=\frac{\log t}{\log q},
$$

up to an additive constant. Hence, the function $P$ finally takes the form

$$
\begin{aligned}
P= & \rho_{0} Q+\alpha \log (t) Q+\left(t-t^{-1}\right)^{+}\left(t-t^{-1}\right)^{-} r+\frac{1}{2}\left(a_{+}-a_{-}\right)\left(t-t^{-1}\right)\left(q^{\frac{1}{2}}-q^{-\frac{1}{2}}\right) \bar{Q} \\
& +\frac{1}{2}\left(a_{+}+a_{-}\right)\left(p_{q}^{++}+p_{q}^{--}\right) Q
\end{aligned}
$$

with $\rho_{0}$ a polynomial, and $\alpha=\frac{\pi_{0}}{\log q}$ a constant, where $\pi_{0}$ is the constant term of $\pi$. For $N$ odd $\alpha$ vanishes [14], but it can be non-zero for $N$ even. Thus we see that even in absence of strings, $P$ is not always a polynomial, but what we will call a 'quasi-polynomial', i.e. a polynomial plus $\log t$ times a polynomial.

Quasi-polynomiality of $P$ (in $t, t^{-1}$ ) requires $a_{+}=-a_{-}$, which is equivalent to the admissibility of the Bethe roots (3.6). As for the polynomiality of the function $Q_{0,2}$ (and $Q_{0, n}$ ), one sees that the $\log$ part of $P^{(n) \pm}$ is $\alpha \log (t) Q^{(n) \pm}$ so that it always cancels out in $Q_{0, n}$. Then one discovers that the polynomiality of the function $Q_{0,2}$ is also equivalent to the $a_{+}=-a_{-}$condition. Thus, (almost) similarly to the XXX case, the following statements are equivalent: (i) $P$ is a quasi-polynomial, (ii) all $Q_{a, s}$ functions are polynomial, (iii) the roots $\left\{u_{1}, \ldots, u_{M}\right\}$ are admissible solutions of the BA equations, (iv) the Bethe vector is an eigenvector of the transfer matrix. 
Let us briefly comment on the root of unity case $q=e^{i \pi / p}$ with integer $p \geq 2$. In this case, another exact string besides $\pm \eta / 2$ becomes possible, namely, a complete string of length $p$ [20-24], due to the periodicity of $\sinh$ in the imaginary direction. Thus the construction of the function $P$ would involve more $q$-deformed digamma functions located at the center of these new exact strings, and the quasi-polynomiality of $P$ would be equivalent to the cancellation of multiple constants. It would a priori require more work to show that their cancellation are equivalent to the $Q Q$-relations, as we should expect.

\subsubsection{An example}

We now present an explicit example of using the Q-system (3.8)-(3.9) to compute Bethe roots. The functions $Q_{0,0}(t)$ and $Q_{1,0}(t)$ are now given by ${ }^{7}$

$\mathrm{Q}\left[0,0, t_{-}\right]:=\left(t-t^{\wedge}(-1)\right)^{\wedge} L$;

$\mathrm{Q}\left[1,0, t_{-}\right]:=t^{\wedge}(-M) / c[0]+\operatorname{Sum}\left[c[k] t^{\wedge}(2 k-M),\{k, 1, M-1\}\right]+t^{\wedge} M$;

and the functions $Q_{1, n}(t)$ and $Q_{0, n}(t)$ are given by

$\begin{aligned} & \mathrm{Q}\left[1, \mathrm{n}_{-}, \mathrm{t}_{-}\right]:=\mathrm{Q}[1, \mathrm{n}-1, \mathrm{t} \operatorname{Exp}[\operatorname{eta} / 2]]-\mathrm{Q}[1, \mathrm{n}-1, \mathrm{t} \operatorname{Exp}[-(\mathrm{eta} / 2)]] ; \\ & \mathrm{Q}[0,\left.\mathrm{n}_{-}, \mathrm{t}_{-}\right]:=(\mathrm{Q}[1, \mathrm{n}, \mathrm{t} \operatorname{Exp}[\operatorname{eta} / 2]] \mathrm{Q}[0, \mathrm{n}-1, \mathrm{Exp}[-(\mathrm{eta} / 2)]]- \\ &\mathrm{Q}[1, \mathrm{n}, \mathrm{t} \operatorname{Exp}[-(\operatorname{eta} / 2)]] \mathrm{Q}[0, \mathrm{n}-1, \mathrm{t} \operatorname{Exp}[\operatorname{eta} / 2]]) / \mathrm{Q}[1, \mathrm{n}-1, \mathrm{t}] ;\end{aligned}$

Moreover, we define $y_{n}(t)$ by

$\mathrm{y}\left[\mathrm{n}_{-}, \mathrm{t}_{-}\right]:=\operatorname{PolynomialRemainder}\left[\operatorname{Numerator}\left[\mathrm{t}^{\wedge} \mathrm{L}\right.\right.$ Together $\left.[\mathrm{Q}[0, \mathrm{n}, \mathrm{t}]]\right]$, Denominator $\left[\mathrm{t}^{\wedge} \mathrm{L}\right.$ Together $\left.\left.[\mathrm{Q}[0, \mathrm{n}, \mathrm{t}]]\right], \mathrm{t}\right]$;

where we have inserted $t^{L}$ factors to ensure that both the numerator and denominator are polynomials in $t$. We then solve for the coefficients $c_{k}$ that make $y_{n}(t)$ vanish for all values of $t$. From experience, it is enough to consider $n=1,2, \ldots, M$.

sol = Solve[Table [CoefficientList $[y[n, t], t]=0,\{n, 1, M\}]$, Table $[c[k],\{k, 0, M-1\}]]$

As before, let us consider the case $N \equiv L=6$ and $M=2$, and we now further set $\eta=\log (2)$. The above code generates 15 solutions, in agreement with (3.7). We can solve for the corresponding Bethe roots similarly as before, for example

NSolve $[(Q[1,0, \operatorname{Exp}[u]] / . \operatorname{sol}[[1]])==0, u] / /$ Simplify // Flatten gives \pm 0.346574 .

\footnotetext{
${ }^{7}$ In order to avoid a vanishing $t^{-M}$ term, we define its coefficient as $1 / c[0]$, and we consider solutions with finite $c[0]$.
} 


\section{Open XXX $Q$-system}

We turn now to the open XXX spin chain of length $N$, with Hamiltonian

$$
\mathbb{H}=\sum_{k=1}^{N-1} \vec{\sigma}_{k} \cdot \vec{\sigma}_{k+1},
$$

which is $\mathrm{SU}(2)$ invariant. After reviewing its BA solution, we propose a corresponding $Q$-system, and argue that all the $Q$ 's are polynomial if and only if the Bethe state is an eigenstate of the transfer matrix.

\subsection{Review of the algebraic BA solution}

The transfer matrix $\mathbb{T}(u)$ is given by $[25]$

$$
\mathbb{T}(u)=\operatorname{tr}_{0} \mathbb{U}_{0}(u), \quad \mathbb{U}_{0}(u)=\mathbb{M}_{0}(u) \widehat{\mathbb{M}}_{0}(u),
$$

where $\mathbb{M}_{0}(u)$ is the monodromy matrix in $(2.3)$, and $\widehat{\mathbb{M}}_{0}(u)$ is given by

$$
\widehat{\mathbb{M}}(u)=\mathbb{R}_{0 N}(u) \cdots \mathbb{R}_{02}(u) \mathbb{R}_{01}(u) .
$$

The $R$-matrix is again given by (2.2). Its boundary equivalent, the $K$-matrix, is the identity in the case considered here. By construction, the open-chain transfer matrix (4.2) has the commutativity property

$$
[\mathbb{T}(u), \mathbb{T}(v)]=0,
$$

and it also has the crossing symmetry

$$
\mathbb{T}(-u)=\mathbb{T}(u) .
$$

The Hamiltonian (4.1) is proportional to $\left.\frac{d \mathbb{T}(u)}{d u}\right|_{u=i / 2}$, up to an additive constant.

We denote the matrix elements of $\mathbb{U}_{0}(u)(4.2)$ as follows

$$
\mathbb{U}_{0}(u)=\left(\begin{array}{cc}
\mathbb{A}(u) & \mathbb{B}(u) \\
\mathbb{C}(u) & \frac{u^{-}}{u} \mathbb{D}(u)+\frac{i}{2 u} \mathbb{A}(u)
\end{array}\right) .
$$

The reference state $(2.5)$ is annihilated by $\mathbb{C}(u)$, and is an eigenstate of $\mathbb{A}(u)$ and $\mathbb{D}(u)$, with

$$
\mathbb{A}(u)|0\rangle=\left(u^{+}\right)^{2 N}|0\rangle, \quad \mathbb{D}(u)|0\rangle=\left(u^{-}\right)^{2 N}|0\rangle .
$$

The Bethe states are defined by

$$
\left|u_{1} \ldots u_{M}\right\rangle=\prod_{k=1}^{M} \mathbb{B}\left(u_{k}\right)|0\rangle .
$$

The Bethe states satisfy (for any $\left\{u_{1}, \ldots, u_{M}\right\}$ ) the off-shell relation

$$
\mathbb{T}(u)\left|u_{1} \ldots u_{M}\right\rangle=T(u)\left|u_{1} \ldots u_{M}\right\rangle+\sum_{j=1}^{M} F_{j}\left|u, u_{1} \ldots \hat{u}_{j} \ldots u_{M}\right\rangle,
$$


where $\hat{u}_{j}$ is omitted. Moreover, $T(u)$ is given by the $T Q$-relation

$$
u T(u) Q(u)=\left(u^{+}\right)^{2 N+1} Q^{--}(u)+\left(u^{-}\right)^{2 N+1} Q^{++}(u), \quad Q(u)=\prod_{k=1}^{M}\left(u-u_{k}\right)\left(u+u_{k}\right)
$$

and the coefficients $F_{j}$ of the "unwanted" terms are given by

$$
\begin{aligned}
F_{j}=\frac{2 i u^{+}\left(u_{j}-\frac{i}{2}\right)}{u_{j}\left(u-u_{j}\right)\left(u+u_{j}\right)}[ & {\left[\left(u_{j}+\frac{i}{2}\right)^{2 N} \prod_{\substack{k \neq j \\
k=1}}^{M} \frac{\left(u_{j}-u_{k}-i\right)\left(u_{j}+u_{k}-i\right)}{\left(u_{j}-u_{k}\right)\left(u_{j}+u_{k}\right)}\right.} \\
& \left.-\left(u_{j}-\frac{i}{2}\right)^{2 N} \prod_{\substack{k \neq j \\
k=1}}^{M} \frac{\left(u_{j}-u_{k}+i\right)\left(u_{j}+u_{k}+i\right)}{\left(u_{j}-u_{k}\right)\left(u_{j}+u_{k}\right)}\right] .
\end{aligned}
$$

We again write $f^{ \pm}(u)=f\left(u \pm \frac{i}{2}\right)$, as in the closed XXX case. Note that both $Q$ and $T$ are even functions of $u$

$$
Q(-u)=Q(u), \quad T(-u)=T(u) .
$$

Substituting $u=u_{j}$ in the $T Q$-relation (4.10), we see that the l.h.s. vanishes, and we obtain

$$
\begin{aligned}
& \left(u_{j}+\frac{i}{2}\right)^{2 N+1} \prod_{k=1}^{M}\left(u_{j}-u_{k}-i\right)\left(u_{j}+u_{k}-i\right)+\left(u_{j}-\frac{i}{2}\right)^{2 N+1} \prod_{k=1}^{M}\left(u_{j}-u_{k}+i\right)\left(u_{j}+u_{k}+i\right)=0, \\
& j=1, \ldots, M .
\end{aligned}
$$

If $u_{j} \neq \pm \frac{i}{2}$, then these equations are equivalent to the BA equations

$$
\left(\frac{u_{j}+\frac{i}{2}}{u_{j}-\frac{i}{2}}\right)^{2 N}=\prod_{\substack{k \neq j \\ k=1}}^{M} \frac{\left(u_{j}-u_{k}+i\right)\left(u_{j}+u_{k}+i\right)}{\left(u_{j}-u_{k}-i\right)\left(u_{j}+u_{k}-i\right)}, \quad j=1, \ldots, M .
$$

The BA equations have the reflection symmetry $u_{j} \mapsto-u_{j}$, while keeping the other $u$ 's (i.e. $u_{k}$ with $k \neq j$ ) unchanged. Hence, without loss of generality, we henceforth assume that $\Re e\left(u_{j}\right)>0$, or $\Re e\left(u_{j}\right)=0$ and $\Im m\left(u_{j}\right) \geq 0$.

Roughly speaking, if $\left\{u_{1}, \ldots, u_{M}\right\}$ satisfy the BA equations (4.14), then all $F_{j}=0$; i.e., the "unwanted" terms in the off-shell relation (4.9) vanish, hence the Bethe state (4.8) is an eigenstate of the transfer matrix, with corresponding eigenvalue $T(u)$. However, there are some important caveats. We argue in appendix A that certain "exceptional" solutions of the BA equations (namely 0 and $\pm \frac{i}{2}$ ) do not lead to eigenstates of the transfer matrix. Moreover, we make the standard assumption (supported by numerical evidence, see e.g. [24]) that the Bethe roots are pairwise distinct, i.e. $u_{j} \neq u_{k}$ if $j \neq k$.

We therefore define an admissible solution $\left\{u_{1}, \ldots, u_{M}\right\}$ of the BA equations (4.14), such that all the $u_{j}$ 's are finite, not equal to $\pm \frac{i}{2}$ or 0 , and pairwise distinct (no two are equal), and each $u_{j}$ satisfies either

$$
\Re e\left(u_{j}\right)>0
$$


or

$$
\Re e\left(u_{j}\right)=0 \quad \text { and } \quad \Im m\left(u_{j}\right)>0 .
$$

The set $\left\{u_{1}, \ldots, u_{M}\right\}$ is an admissible solution of the BA equations if and only if the Bethe state $\left|u_{1} \ldots u_{M}\right\rangle$ is an eigenstate of the transfer matrix $\mathbb{T}(u)$. We emphasize that, for the open XXX chain, there are no physical singular solutions of the Bethe equations - all the singular solutions are unphysical. The number $\mathcal{N}(N, M)$ of admissible solutions of the BA equations with $M \leq N / 2$ has been conjectured [24] to be given by

$$
\mathcal{N}(N, M)=\left(\begin{array}{l}
N \\
M
\end{array}\right)-\left(\begin{array}{c}
N \\
M-1
\end{array}\right)
$$

when $q=e^{\eta}$ is not a root of unity.

\section{$4.2 \quad Q$-system}

We propose the following $Q$-system

$$
u Q_{a+1, s}(u) Q_{a, s+1}(u) \propto Q_{a+1, s+1}^{+}(u) Q_{a, s}^{-}(u)-Q_{a+1, s+1}^{-}(u) Q_{a, s}^{+}(u),
$$

where the nontrivial $Q$-functions for given values of $N$ and $M$ are again defined on the Young diagram in figure 1 , with the boundary conditions $Q_{2, s}=1, Q_{1, s \geq M}=1$, and with the initial condition

$$
Q_{0,0}(u)=u^{2 N} \quad \text { with } \quad Q_{1,0}(u)=Q(u)=\prod_{k=1}^{M}\left(u-u_{k}\right)\left(u+u_{k}\right) .
$$

In contrast to the $Q$-system for periodic XXX (2.13), there is an extra factor of $u$ on the 1.h.s. of (4.18), and the $Q$ 's are even functions of $u$. The degree of the polynomial $Q_{a, s}(u)$ is doubled with respect to the periodic XXX case (namely, twice the number of boxes in the Young diagram to the right and top of the vertex $(a, s)) .{ }^{8}$ We claim that all the $Q$ 's are polynomial if and only if the Bethe state $\left|u_{1} \ldots u_{M}\right\rangle$ (4.8) is an eigenstate of the transfer matrix $\mathbb{T}(u)(4.2)$.

Before entering into the proof, let us quickly check that this $Q$-system indeed leads to the correct BA equations for $\left\{u_{1}, \ldots, u_{M}\right\}$. We write the $Q Q$-equations for $(a, s)=(0,0)$ :

$$
u Q_{1,0} Q_{0,1} \propto Q_{1,1}^{+} Q_{0,0}^{-}-Q_{1,1}^{-} Q_{0,0}^{+},
$$

and for $(a, s)=(1,0)$ :

$$
u Q_{2,0} Q_{1,1} \propto Q_{2,1}^{+} Q_{1,0}^{-}-Q_{2,1}^{-} Q_{1,0}^{+} .
$$

Since $Q_{2,0}=Q_{2,1}=1$, the latter reduces to

$$
u Q_{1,1} \propto Q^{-}-Q^{+} .
$$

Performing the shifts $u \mapsto u \pm \frac{i}{2}$ in (4.22) and evaluating at $u=u_{j}$, we obtain

$$
\left(u_{j}+\frac{i}{2}\right) Q_{1,1}^{+}\left(u_{j}\right) \propto-Q^{++}\left(u_{j}\right), \quad\left(u_{j}-\frac{i}{2}\right) Q_{1,1}^{-}\left(u_{j}\right) \propto Q^{--}\left(u_{j}\right)
$$

\footnotetext{
${ }^{8}$ Equivalently, the Q-functions are polynomials in the variable $u^{2}$ of the same degree as in the periodic XXX case.
} 
since $Q\left(u_{j}\right)=0$. Moreover, evaluating (4.20) at $u=u_{j}$ gives

$$
Q_{1,1}^{+}\left(u_{j}\right) Q_{0,0}^{-}\left(u_{j}\right)=Q_{1,1}^{-}\left(u_{j}\right) Q_{0,0}^{+}\left(u_{j}\right) .
$$

Substituting (4.23) into the above relation gives

$$
-\left(u_{j}-\frac{i}{2}\right) Q^{++}\left(u_{j}\right) Q_{0,0}^{-}\left(u_{j}\right)=\left(u_{j}+\frac{i}{2}\right) Q^{--}\left(u_{j}\right) Q_{0,0}^{+}\left(u_{j}\right),
$$

which coincides with the BA equations (4.13).

\subsection{1 $Q_{a, s}$ in terms of $Q$ and $P$}

We now solve the $Q$-system (4.18) in terms of $Q(u)$ and a function $P(u)$, such that polynomiality of $P(u)$ implies polynomiality of all the $Q$ 's. We define $P(u)$ by $^{9}$

$$
P^{+} Q^{-}-P^{-} Q^{+}=u Q_{0,0},
$$

where $Q_{0,0}$ is given by (4.19). It follows from (4.20) and (4.22) that

$$
Q_{1,1} \propto \frac{Q^{\prime}}{u}=D Q, \quad u Q_{0,1} \propto(D P)^{+}(D Q)^{-}-(D P)^{-}(D Q)^{+},
$$

where we have used the following compact notation for discrete derivatives with certain $1 / u$ factors

$$
\begin{aligned}
D f & =\frac{1}{u}\left(f^{+}-f^{-}\right)=\frac{f^{\prime}}{u}, \\
D^{2} f & =\frac{1}{u}\left[(D f)^{+}-(D f)^{-}\right], \ldots \\
D^{n} f & =\frac{1}{u}\left[\left(D^{n-1} f\right)^{+}-\left(D^{n-1} f\right)^{-}\right] .
\end{aligned}
$$

Similarly, we obtain

$$
Q_{1,2} \propto D^{2} Q, \quad u Q_{0,2} \propto\left(D^{2} P\right)^{+}\left(D^{2} Q\right)^{-}-\left(D^{2} P\right)^{-}\left(D^{2} Q\right)^{+},
$$

and in general

$$
Q_{1, n} \propto D^{n} Q, \quad u Q_{0, n} \propto\left(D^{n} P\right)^{+}\left(D^{n} Q\right)^{-}-\left(D^{n} P\right)^{-}\left(D^{n} Q\right)^{+} .
$$

Since both $Q(u)$ and $P(u)$ are even functions of $u$, it follows that $D Q$ and $D P$ are also even functions of $u$. Hence, if $P(u)$ is a polynomial function of $u$, then the r.h.s. of the second equation in (4.27) is divisible by $u$, thus $Q_{0,1}$ is polynomial; and, from (4.30), we similarly conclude that all the $Q$ 's are polynomial.

We observe, similarly to the closed-chain case, that the $T Q$-equation (4.10) together with the definition of $P(4.26)$ imply

$$
u T=P^{++} Q^{--}-P^{--} Q^{++} .
$$

It follows that $P$ is also a solution of the $T Q$-equation

$$
u T P=\left(u^{+}\right)^{2 N+1} P^{--}+\left(u^{-}\right)^{2 N+1} P^{++} .
$$

Hence, (4.26) can be regarded as the Wronskian relation obeyed by the two solutions $Q$ and $P$ of the $T Q$-equation (4.10).

\footnotetext{
${ }^{9}$ As in the closed-chain case, for given $Q$, this equation does not uniquely define $P$ : if $P(u)$ is a solution of (4.26), then so is $P(u)+\alpha Q(u)$, for any constant value of $\alpha$.
} 


\subsubsection{Construction of $P$}

We now construct the function $P(u)$ for a set $\left\{u_{1}, \ldots, u_{M}\right\}$, and argue that $P(u)$ is polynomial if and only if $\left\{u_{1}, \ldots, u_{M}\right\}$ is an admissible solution of the BA equations.

The construction of the $P$-function for the open chain is similar to that for the closed chain, but with some significant differences. In the presence of one singular root $\frac{i}{2}$ and one zero root 0 , the $Q$-function takes the form ${ }^{10}$

$$
Q(u)=u^{+} u^{-} u^{2} \bar{Q}(u), \quad \bar{Q}(u)=\prod_{\substack{u_{k} \neq \frac{i}{2}, 0 \\ k=1}}^{M}\left(u-u_{k}\right)\left(u+u_{k}\right) .
$$

We define the function $R(u)$

$$
R=\frac{u^{2 N+1}}{Q^{+} Q^{-}}=\frac{u^{2 N-1}}{u^{++} u^{--}\left(u^{+} u^{-}\right)^{2} \bar{Q}^{+} \bar{Q}^{-}},
$$

which is related to $P(u)$ defined in $(4.26)$ by

$$
R=\left(\frac{P}{Q}\right)^{\prime}=\frac{P^{+}}{Q^{+}}-\frac{P^{-}}{Q^{-}} .
$$

Decomposing (4.34) in partial fractions, we obtain

$$
R=\pi+\frac{q_{+}}{\bar{Q}^{+}}+\frac{q_{-}}{\bar{Q}^{-}}+\frac{a_{+}}{u^{++}}+\frac{b_{+}}{u^{+}}+\frac{c_{+}}{\left(u^{+}\right)^{2}}+\frac{a_{-}}{u^{--}}+\frac{b_{-}}{u^{-}}+\frac{c_{-}}{\left(u^{-}\right)^{2}},
$$

where $\pi$ is a polynomial of order $2 N-4 M+1, q_{ \pm}$are polynomials of degree less than that of $\bar{Q}$, and $a_{ \pm}, b_{ \pm}, c_{ \pm}$are constants. Note that $a_{ \pm}$arise from the presence of the singular root, while $b_{ \pm}, c_{ \pm}$are due to the presence of the zero root. From the TQ-relation (4.10), we obtain

$$
R^{+}+R^{-}=\frac{u T}{Q^{++} Q^{--}}=\frac{u T}{u^{+} u^{-} u^{+++} u^{---}\left(u^{++} u^{--}\right)^{2} \bar{Q}^{++} \bar{Q}^{--}} .
$$

We now evaluate the l.h.s. of (4.37) using (4.36), and consider the values of $u$ where singularities could arise. The r.h.s. of (4.37) has no singularities at the zeros of $\bar{Q}$ (recall that $T(u)$ is regular for values of $u$ corresponding to admissible Bethe roots), hence

$$
q_{+}=q^{+}, \quad q_{-}=-q^{-},
$$

for some polynomial $q(u)$. From the residues of (4.37) at $u=\mp \frac{i}{2}$, we obtain

$$
a_{ \pm}=\frac{4 T\left(\mp \frac{i}{2}\right)}{9 \bar{Q}\left( \pm \frac{i}{2}\right) \bar{Q}\left(\mp \frac{3 i}{2}\right)}=\frac{8(-1)^{N+1}}{9 \bar{Q}\left( \pm \frac{i}{2}\right) \bar{Q}\left(\mp \frac{3 i}{2}\right)} .
$$

Since $Q(u)$ is an even function of $u$, we conclude - in significant contrast from the closedchain case - that $a_{+}=a_{-} \equiv a$. From the residues of (4.37) at $u=\mp i$, we can obtain expressions for $c_{ \pm}$and $b_{ \pm}$, and we find that

$$
c \equiv c_{+}=-c_{-}, \quad b \equiv b_{+}=b_{-},
$$

\footnotetext{
${ }^{10}$ The cases of either one singular root or one zero root are essentially special cases, for which $b_{ \pm}=c_{ \pm}=0$ or $a_{ \pm}=0$ in (4.36) below, respectively.
} 
which is consistent with the constraints coming from the residues of (4.37) at $u=0$ (note that the presence of a zero root implies that $T(u)$ has a double pole at $u=0$ ).

We write the polynomial $\pi$ in (4.36) as

$$
\pi=\rho^{\prime}=\rho^{+}-\rho^{-},
$$

where $\rho$ is a polynomial. Recalling the definition of the function $p(u)(2.33)$, we see that

$$
R=\left(\rho+\frac{q}{\bar{Q}}+a\left(p^{++}+p^{--}\right)+b\left(p^{+}+p^{-}\right)+\frac{c}{u^{2}}\right)^{\prime} .
$$

It immediately follows from (4.35) that the $P$-function is given by

$$
P=\rho Q+u^{+} u^{-} u^{2} q+c u^{+} u^{-} \bar{Q}+a\left(p^{++}+p^{--}\right) Q+b\left(p^{+}+p^{-}\right) Q,
$$

which is a polynomial iff $a=b=0$. That is, the $P$-function is polynomial iff there is no singular root and no zero root, in which case $\left\{u_{1}, \ldots, u_{M}\right\}$ is admissible. Moreover, $Q_{0,2}$ is polynomial iff $a=b=0$, as follows from a similar analysis to the periodic case in section 2.2.2.

The proof of the $Q$-system (4.18) is now complete, since we have argued as before that the following statements are equivalent: (i) $P$ is a polynomial, (ii) all $Q_{a, s}$-functions are polynomial, (iii) the roots $\left\{u_{1}, \ldots, u_{M}\right\}$ are admissible solutions of the BA equations, (iv) the Bethe vector is an eigenvector of the transfer matrix.

\subsubsection{An example}

We now present an explicit example of using the Q-system (4.18)-(4.19) to compute Bethe roots. The code is similar to the one in section 2.2.3. The functions $Q_{0,0}(u)$ and $Q_{1,0}(u)$ are now given by

$\mathrm{Q}\left[0,0, \mathrm{u}_{-}\right]:=\mathrm{u}^{\wedge}(2 \mathrm{~L})$;

$Q\left[1,0, u_{-}\right]:=\operatorname{Sum}\left[c[k] u^{\wedge}(2 k),\{k, 0, M-1\}\right]+u^{\wedge}(2 M)$;

while $Q_{1, n}(u)$ and $Q_{0, n}(u)$ are given by

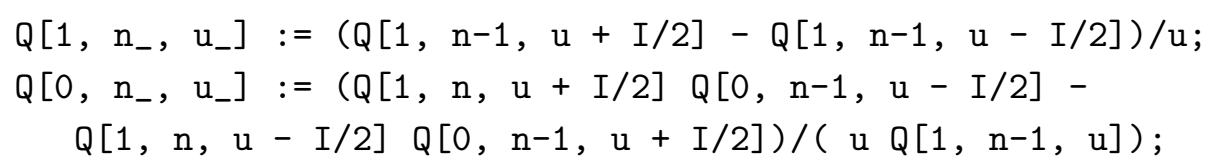

We define $y_{n}(u)$ and solve for the coefficients $c_{k}$ exactly as in section 2.2.3.

For the case $N \equiv L=6, M=2$, the above code generates 9 solutions, in agreement with (4.17). For example, for the first solution, the corresponding Bethe roots are given by $0.301932,1.26627$.

\section{Open quantum-group-invariant XXZ $Q$-system}

We now generalize the preceding results to the open quantum-group-invariant XXZ spin chain, whose Hamiltonian is given by [9]

$$
\mathbb{H}=\sum_{k=1}^{N-1}\left[\sigma_{k}^{x} \sigma_{k+1}^{x}+\sigma_{k}^{y} \sigma_{k+1}^{y}+\frac{1}{2}\left(q+q^{-1}\right) \sigma_{k}^{z} \sigma_{k+1}^{z}\right]-\frac{1}{2}\left(q-q^{-1}\right)\left(\sigma_{1}^{z}-\sigma_{N}^{z}\right) .
$$

For simplicity, we restrict to generic values of $q=e^{\eta}$. 


\subsection{Review of the algebraic BA solution}

The transfer matrix is now given by [25]

$$
\mathbb{T}(u)=\operatorname{tr}_{0} \mathbb{K}_{0}^{L}(u) \mathbb{U}_{0}(u), \quad \mathbb{U}_{0}(u)=\mathbb{M}_{0}(u) \mathbb{K}_{0}^{R}(u) \widehat{\mathbb{M}}_{0}(u),
$$

where the $R$-matrix is again given by (3.2), and the left and right $K$-matrices (solutions of boundary Yang-Baxter equations) are given by the diagonal matrices

$$
\mathbb{K}^{L}(u)=\operatorname{diag}\left(e^{-u-\frac{\eta}{2}}, e^{u+\frac{\eta}{2}}\right), \quad \mathbb{K}^{R}(u)=\operatorname{diag}\left(e^{u-\frac{\eta}{2}}, e^{-u+\frac{\eta}{2}}\right) .
$$

The transfer matrix (5.2) has the commutativity property (4.4) as well as the crossing symmetry (4.5). The Hamiltonian (5.1) is proportional to $d \mathbb{T}(u) /\left.d u\right|_{u=\eta / 2}$, up to an additive constant.

We define the elements of $\mathbb{U}_{0}(u)(5.2)$ as follows

$$
\mathbb{U}_{0}(u)=\left(\begin{array}{cc}
e^{u-\frac{\eta}{2}} \mathbb{A}(u) & \mathbb{B}(u) \\
\mathbb{C}(u) & \frac{e^{-u-\frac{\eta}{2}} \sinh (2 u-\eta)}{\sinh (2 u)} \mathbb{D}(u)+\frac{e^{u-\frac{\eta}{2}} \sinh (\eta)}{\sinh (2 u)} \mathbb{A}(u)
\end{array}\right) .
$$

The reference state $(2.5)$ is annihilated by $\mathbb{C}(u)$, and is an eigenstate of $\mathbb{A}(u)$ and $\mathbb{D}(u)$

$$
\mathbb{A}(u)|0\rangle=\sinh ^{2 N}\left(u+\frac{\eta}{2}\right)|0\rangle, \quad \mathbb{D}(u)|0\rangle=\sinh ^{2 N}\left(u-\frac{\eta}{2}\right)|0\rangle .
$$

The Bethe states are again defined by

$$
\left|u_{1} \ldots u_{M}\right\rangle=\prod_{k=1}^{M} \mathbb{B}\left(u_{k}\right)|0\rangle .
$$

The off-shell equation is

$$
\mathbb{T}(u)\left|u_{1} \ldots u_{M}\right\rangle=T(u)\left|u_{1} \ldots u_{M}\right\rangle+\sum_{j=1}^{M} F_{j}\left|u, u_{1} \ldots \hat{u}_{j} \ldots u_{M}\right\rangle,
$$

where $T(u)$ is given by the $T Q$-relation

$$
\begin{aligned}
\sinh (2 u) T(u) Q(u)= & \sinh (2 u+\eta) \sinh ^{2 N}\left(u+\frac{\eta}{2}\right) Q(u-\eta) \\
& +\sinh (2 u-\eta) \sinh ^{2 N}\left(u-\frac{\eta}{2}\right) Q(u+\eta),
\end{aligned}
$$

with

$$
Q(u)=\prod_{k=1}^{M} \sinh \left(u-u_{k}\right) \sinh \left(u+u_{k}\right),
$$

and $F_{j}$ is given by

$$
\begin{aligned}
F_{j}= & \frac{\sinh (2 u+\eta) \sinh \left(2 u_{j}-\eta\right) \sinh (\eta)}{\sinh \left(2 u_{j}\right) \sinh \left(u-u_{j}\right) \sinh \left(u+u_{j}\right)} \\
\times & {\left[\sinh ^{2 N}\left(u_{j}+\frac{\eta}{2}\right) \prod_{\substack{k \neq j \\
k=1}}^{M} \frac{\sinh \left(u_{j}-u_{k}-\eta\right) \sinh \left(u_{j}+u_{k}-\eta\right)}{\sinh \left(u_{j}-u_{k}\right) \sinh \left(u_{j}+u_{k}\right)}\right.} \\
& \left.\quad-\sinh ^{2 N}\left(u_{j}-\frac{\eta}{2}\right) \prod_{\substack{k \neq j \\
k=1}}^{M} \frac{\sinh \left(u_{j}-u_{k}+\eta\right) \sinh \left(u_{j}+u_{k}+\eta\right)}{\sinh \left(u_{j}-u_{k}\right) \sinh \left(u_{j}+u_{k}\right)}\right] .
\end{aligned}
$$


Substituting $u=u_{j}$ in the $T Q$-equation (5.8), we see that the l.h.s. vanishes, and we obtain

$$
\begin{aligned}
& \sinh \left(2 u_{j}+\eta\right) \sinh ^{2 N}\left(u_{j}+\frac{\eta}{2}\right) \prod_{k=1}^{M} \sinh \left(u_{j}-u_{k}-\eta\right) \sinh \left(u_{j}+u_{k}-\eta\right) \\
& +\sinh \left(2 u_{j}-\eta\right) \sinh ^{2 N}\left(u_{j}-\frac{\eta}{2}\right) \prod_{k=1}^{M} \sinh \left(u_{j}-u_{k}+\eta\right) \sinh \left(u_{j}+u_{k}+\eta\right)=0, \quad j=1, \ldots, M
\end{aligned}
$$

If $u_{j} \neq \pm \frac{\eta}{2}$, then these equations are equivalent to the BA equations

$$
\left(\frac{\sinh \left(u_{j}+\frac{\eta}{2}\right)}{\sinh \left(u_{j}-\frac{\eta}{2}\right)}\right)^{2 N}=\prod_{\substack{k \neq j \\ k=1}}^{M} \frac{\sinh \left(u_{j}-u_{k}+\eta\right) \sinh \left(u_{j}+u_{k}+\eta\right)}{\sinh \left(u_{j}-u_{k}-\eta\right) \sinh \left(u_{j}+u_{k}-\eta\right)}, \quad j=1, \ldots, M .
$$

The BA equations have the reflection symmetry $u_{j} \mapsto-u_{j}$ (while keeping the other $u$ 's unchanged), as well as the periodicity $u_{j} \mapsto u_{j}+i \pi$.

We must exclude solutions with roots $0, \pm \frac{i \pi}{2}$ and $\pm \frac{\eta}{2}$, see section A.2. We therefore define an admissible solution $\left\{u_{1}, \ldots, u_{M}\right\}$ of the BA equations (5.12), such that all the $u_{j}$ 's are finite, not equal to $0, \pm \frac{i \pi}{2}$ or $\pm \frac{\eta}{2}$, pairwise distinct, and each $u_{j}$ satisfies either

$$
\Re e\left(u_{j}\right)>0 \quad \text { and } \quad-\frac{\pi}{2}<\Im m\left(u_{j}\right) \leq \frac{\pi}{2}
$$

or

$$
\Re e\left(u_{j}\right)=0 \quad \text { and } \quad 0<\Im m\left(u_{j}\right)<\frac{\pi}{2} .
$$

The set $\left\{u_{1}, \ldots, u_{M}\right\}$ is an admissible solution of the BA equations if and only if the Bethe state $\left|u_{1} \ldots u_{M}\right\rangle$ is an eigenstate of the transfer matrix $\mathbb{T}(u)$. The number $\mathcal{N}(N, M)$ of admissible solutions of the BA equations with $M \leq N / 2$ has been conjectured [24] to be given again by (4.17).

\section{$5.2 \quad Q$-system}

We propose the following $Q$-system ${ }^{11}$

$$
\left(t^{2}-t^{-2}\right) Q_{a+1, s}(t) Q_{a, s+1}(t) \propto Q_{a+1, s+1}^{+}(t) Q_{a, s}^{-}(t)-Q_{a+1, s+1}^{-}(t) Q_{a, s}^{+}(t),
$$

where the nontrivial $Q$-functions for given values of $N$ and $M$ are again defined on the Young diagram in figure 1 , with the boundary conditions $Q_{2, s}=1, Q_{1, s \geq M}=1$, and with the initial condition

$$
Q_{0,0}(t)=\left(t-t^{-1}\right)^{2 N} \quad \text { with } \quad Q_{1,0}(t)=Q(t)=\prod_{k=1}^{M}\left(t t_{k}^{-1}-t^{-1} t_{k}\right)\left(t t_{k}-t^{-1} t_{k}^{-1}\right) .
$$

In contrast to the periodic XXZ case (3.8), there is an extra factor $\left(t^{2}-t^{-2}\right) \propto \sinh (2 u)$ on the l.h.s. of (5.15). This $Q$-system indeed leads to the BA equations (5.11), as can be seen by following the same logic (4.20)-(4.25) of the rational case. The degree of the polynomials (highest power of $t \equiv e^{u}$ ) is the same as in the open XXX case.

\footnotetext{
${ }^{11}$ As for the periodic XXZ case, here again $t=e^{u}$, and $f^{ \pm}(t)=f\left(t q^{ \pm \frac{1}{2}}\right)$.
} 


\subsection{1 $Q_{a, s}$ in terms of $Q$ and $P$}

We now define $P$ by

$$
P^{+} Q^{-}-P^{-} Q^{+}=\left(t^{2}-t^{-2}\right) Q_{0,0},
$$

where $Q_{0,0}$ is given by (5.16). Similarly to the rational case (4.30), we find that the $Q$ system (5.15) implies that

$$
Q_{1, n} \propto D^{n} Q, \quad\left(t^{2}-t^{-2}\right) Q_{0, n} \propto\left(D^{n} P\right)^{+}\left(D^{n} Q\right)^{-}-\left(D^{n} P\right)^{-}\left(D^{n} Q\right)^{+},
$$

where $D$ is now defined by

$$
\begin{aligned}
D f & =\frac{1}{\left(t^{2}-t^{-2}\right)}\left(f^{+}-f^{-}\right)=\frac{f^{\prime}}{\left(t^{2}-t^{-2}\right)}, \\
D^{2} f & =\frac{1}{\left(t^{2}-t^{-2}\right)}\left[(D f)^{+}-(D f)^{-}\right], \ldots \\
D^{n} f & =\frac{1}{\left(t^{2}-t^{-2}\right)}\left[\left(D^{n-1} f\right)^{+}-\left(D^{n-1} f\right)^{-}\right],
\end{aligned}
$$

cf. (4.28). Note that $P$ is also a solution of the $T Q$-relation (5.8)

$$
\sinh (2 u) T P=\sinh (2 u+\eta) Q_{0,0}^{+} P^{--}+\sinh (2 u-\eta) Q_{0,0}^{-} P^{++},
$$

cf. (4.32).

\subsubsection{Construction of $P$}

The construction of $P$ parallels the rational case. In the presence of one singular root $\left(u=\frac{\eta}{2}, t=q^{\frac{1}{2}}\right)$ and one zero root $\left(u=0, t=1\right.$; the case $u=\frac{i \pi}{2}, t=-1$ is similar $)$, the $Q$-function becomes

$$
Q(t)=\left(t-t^{-1}\right)^{+}\left(t-t^{-1}\right)^{-}\left(t-t^{-1}\right)^{2} \bar{Q}(t)
$$

We now define $R(t)$ as

$$
R(t)=\frac{\left(t^{2}-t^{-2}\right)\left(t-t^{-1}\right)^{2 N}}{Q^{+} Q^{-}}=\frac{\left(t^{2}-t^{-2}\right)\left(t-t^{-1}\right)^{2 N-2}}{\left(t-t^{-1}\right)^{++}\left(t-t^{-1}\right)^{--}\left[\left(t-t^{-1}\right)^{+}\left(t-t^{-1}\right)^{-}\right]^{2} \bar{Q}^{+} \bar{Q}^{-}},
$$

which is related to $P(5.17)$ by $R=\left(\frac{P}{Q}\right)^{\prime}$. We decompose $R$ as follows

$$
\begin{aligned}
R= & \pi+\frac{r_{+}}{\bar{Q}^{+}}+\frac{r_{-}}{\bar{Q}^{-}}+\frac{a_{+}}{\left(t-t^{-1}\right)^{++}}+\frac{b_{+}}{\left(t-t^{-1}\right)^{+}}+\frac{c_{+}}{\left[\left(t-t^{-1}\right)^{+}\right]^{2}} \\
& +\frac{a_{-}}{\left(t-t^{-1}\right)^{--}}+\frac{b_{-}}{\left(t-t^{-1}\right)^{-}}+\frac{c_{-}}{\left[\left(t-t^{-1}\right)^{-}\right]^{2}},
\end{aligned}
$$

where $\pi$ is polynomial. From the $T Q$-relation (5.8), we obtain

$$
\begin{aligned}
R^{+}+R^{-} & =\frac{2^{2 N}\left(t^{2}-t^{-2}\right) T}{Q^{++} Q^{--}} \\
& =\frac{2^{2 N}\left(t^{2}-t^{-2}\right) T}{\left(t-t^{-1}\right)^{+}\left(t-t^{-1}\right)^{-}\left(t-t^{-1}\right)^{+++}\left(t-t^{-1}\right)^{---}\left[\left(t-t^{-1}\right)^{++}\left(t-t^{-1}\right)^{--}\right]^{2} \bar{Q}^{++} \bar{Q}^{--}} .
\end{aligned}
$$


From the singularity structure of this equation and the fact that $Q(t)$ and $T(t)$ are invariant under $t \mapsto t^{-1}$, we again obtain

$$
r_{+}=r^{+}, \quad r_{-}=-r^{-},
$$

where $r(t)$ is a polynomial in $t$ and $t^{-1}$, and

$$
a_{+}=a_{-} \equiv a, \quad c \equiv c_{+}=-c_{-}, \quad b \equiv b_{+}=b_{-} .
$$

The expression (5.23) for $R$ can therefore be rewritten in the form

$$
R=\left(\rho+\frac{r}{\bar{Q}}+a\left(p_{q}^{++}+p_{q}^{--}\right)+b\left(p_{q}^{+}+p_{q}^{-}\right)+\frac{c}{\left(t-t^{-1}\right)^{2}}\right)^{\prime},
$$

where $p_{q}(t)$ is defined in (3.16), cf. (4.42), and $\rho^{\prime}=\pi .{ }^{12}$ Since $R=\left(\frac{P}{Q}\right)^{\prime}$, we conclude that $P$ is given by

$$
\begin{aligned}
P=\rho Q & +\left(t-t^{-1}\right)^{+}\left(t-t^{-1}\right)^{-}\left(t-t^{-1}\right)^{2} r+c\left(t-t^{-1}\right)^{+}\left(t-t^{-1}\right)^{-} \bar{Q} \\
& +a\left(p_{q}^{++}+p_{q}^{--}\right) Q+b\left(p_{q}^{+}+p_{q}^{-}\right) Q,
\end{aligned}
$$

which is a polynomial in $t$ and $t^{-1}$ iff $a=b=0$. That is, as in the rational case, $P$ is polynomial iff there is no singular root and no zero root, in which case $\left\{u_{1}, \ldots, u_{M}\right\}$ is admissible. Moreover, $Q_{0,2}$ is polynomial iff $a=b=0$.

\subsubsection{An example}

Let us finally present an explicit example of using the Q-system (5.15)-(5.16) to compute Bethe roots. The functions $Q_{0,0}(t)$ and $Q_{1,0}(t)$ are now given by

$Q\left[0,0, t_{-}\right]:=\left(t-t^{\wedge}(-1)\right)^{\wedge}(2 L)$;

$Q\left[1,0, t_{-}\right]:=\operatorname{Sum}\left[c[k]\left(t^{\wedge}(2 k)+t^{\wedge}(-2 k)\right),\{k, 0, M-1\}\right]+t^{\wedge}(2 M)+t^{\wedge}(-2 M)$;

and the functions $Q_{1, n}(t)$ and $Q_{0, n}(t)$ are given by

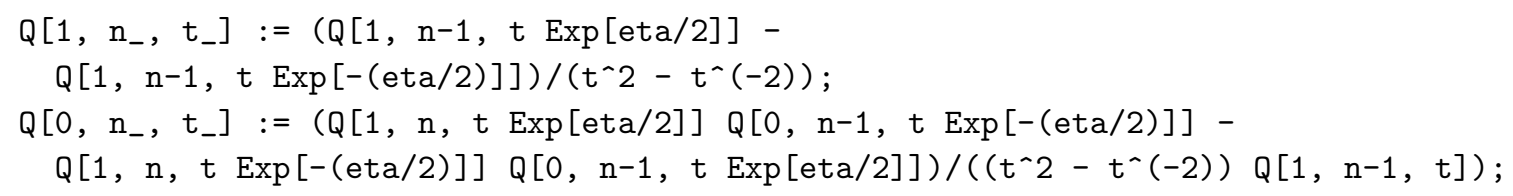

Moreover, we now define $y_{n}(t)$ by

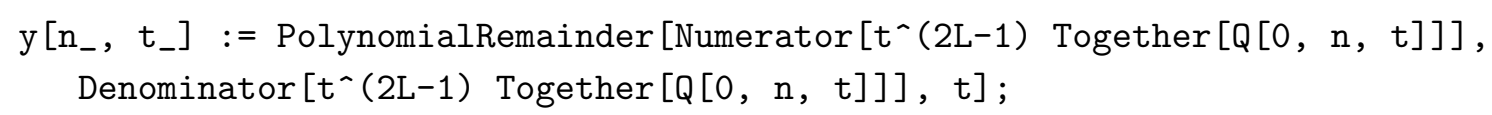

We then solve for the coefficients $c_{k}$ and the Bethe roots exactly as in section 3.2.1.

As before, let us consider the case $N \equiv L=6$ and $M=2$, with $\eta=\log (2)$. The above code generates 9 solutions, as expected (4.17). For example, for the first solution, the corresponding Bethe roots are given by $0.0967267 i, 0.385801 i$.

\footnotetext{
${ }^{12}$ In contrast with the closed XXZ case, a term in $P$ of the form $\alpha \log (t) Q$ as in (3.20) is absent.
} 


\section{Conclusions}

Our main results are $Q$-systems for the closed XXZ (3.8)-(3.9), open XXX (4.18)-(4.19) and open quantum-group-invariant XXZ (5.15)-(5.16) quantum spin chains. Polynomial solutions of these $Q$-systems can be found efficiently, which in turn lead directly to the admissible solutions of the corresponding BA equations.

Numerous applications of these results are possible. In conjunction with techniques from algebraic geometry, these $Q$-systems allow the exact computation of partition functions for trigonometric vertex models and for vertex models with boundaries $[6,7]$.

We restricted here to open spin chains with $\mathrm{SU}(2)$ or $U_{q}(\mathrm{SU}(2))$ symmetry. It would be both interesting and useful to formulate $Q$-systems for open spin chains with other integrable boundary conditions, as well as for integrable models based on $R$-matrices for higher-rank algebras and/or higher-dimensional representations.

\section{Acknowledgments}

ZB and RN are grateful for the warm hospitality extended to them at the University of Miami and the Wigner Research Center, respectively. ZB was supported in part by NKFIH grant K116505. The work of EG and JLJ was supported by the European Research Council under the Advanced Grant NuQFT. RN was supported in part by a Cooper fellowship.

\section{A Exceptional solutions for open spin chains}

\section{A.1 XXX}

\section{A.1.1 $\quad u_{1}=0$}

Let us consider a solution $u_{1}, \ldots, u_{M}$ of the BA equations (4.14) with one zero Bethe root, say $u_{1}=0$ (and $u_{2}, \ldots, u_{M}$ pairwise distinct and not equal to 0 ). Since $F_{1}$ in (4.11) is not well-defined, we set $u_{1}=\epsilon$ and consider the limit $\lim _{\epsilon \rightarrow 0} F_{1}$. It is straightforward to see that this limit exists and is nonzero. Hence, the corresponding Bethe state in the off-shell equation (4.9) is not an eigenstate of the transfer matrix, see also [26].

\section{A.1.2 $\quad u_{1}=\frac{i}{2}$}

The equations (4.13) evidently have solutions $u_{1}, \ldots, u_{M}$ with one "singular" root, say $u_{1}=\frac{i}{2}$ (and $u_{2}, \ldots, u_{M}$ pairwise distinct and not equal to $\pm \frac{i}{2}$ ). However, the BA equations (4.14) do not have such solutions (recall that the latter equations are not equivalent to (4.13) for this case). Hence, it is not surprising that the corresponding Bethe state is not an eigenstate of the transfer matrix. Indeed, let us define a renormalized B-operator

$$
\tilde{\mathbb{B}}(u)=\frac{1}{u^{-}} \mathbb{B}(u),
$$


such that $\lim _{\epsilon \rightarrow 0} \tilde{\mathbb{B}}\left(\frac{i}{2}+\epsilon\right)$ is finite and non-singular. ${ }^{13}$ Bethe states created with this renormalized operator satisfy an off-shell relation similar to (4.9), except with $F_{j}$ replaced by

$$
\tilde{F}_{j}=\frac{u^{-}}{u_{j}-\frac{i}{2}} F_{j}
$$

We find that $\lim _{\epsilon \rightarrow 0} \tilde{F}_{1}$ exists and is nonzero for $u_{1}= \pm \frac{i}{2}+\epsilon$. Hence, the corresponding Bethe state is not an eigenstate of the transfer matrix.

The BA equations (4.14) do have solutions with a pair of singular Bethe roots, e.g. $u_{1}=\frac{i}{2}$ and $u_{2}= \pm \frac{i}{2}$, which must be discarded since $\left|u_{1}\right|$ and $\left|u_{2}\right|$ are not distinct.

\section{A.2 XXZ}

For the XXZ case, the coefficients $F_{j}$ of the "unwanted" terms are given by (5.10). We exclude both $u_{j}=0$ and $u_{j}=\frac{i \pi}{2}$, since (similarly to section A.1.1) the $\operatorname{limit}_{\epsilon \rightarrow 0} F_{1}$ exists and is nonzero for both $u_{1}=\epsilon$ and $u_{1}=\frac{i \pi}{2}+\epsilon$. We must also exclude $u_{j}= \pm \frac{\eta}{2}$ : similarly to section A.1.2, we renormalize the B-operator

$$
\tilde{\mathbb{B}}(u)=\frac{1}{\sinh (2 u-\eta)} \mathbb{B}(u),
$$

so that $\lim _{\epsilon \rightarrow 0} \tilde{\mathbb{B}}\left(\frac{\eta}{2}+\epsilon\right)$ is finite and non-singular. The coefficients of the "unwanted" terms become

$$
\tilde{F}_{j}=\frac{\sinh (2 u-\eta)}{\sinh \left(2 u_{j}-\eta\right)} F_{j}
$$

Then $\lim _{\epsilon \rightarrow 0} \tilde{F}_{1}$ exists and is nonzero for $u_{1}= \pm \frac{\eta}{2}+\epsilon$.

Open Access. This article is distributed under the terms of the Creative Commons Attribution License (CC-BY 4.0), which permits any use, distribution and reproduction in any medium, provided the original author(s) and source are credited.

\section{References}

[1] R. Hagemans and J.-S. Caux, Deformed strings in the Heisenberg model, J. Phys. A 40 (2007) 14605 [arXiv:0707.2803].

[2] W. Hao, R.I. Nepomechie and A.J. Sommese, Completeness of solutions of Bethe's equations, Phys. Rev. E 88 (2013) 052113 [arXiv:1308.4645] [INSPIRE].

[3] C. Marboe and D. Volin, Fast analytic solver of rational Bethe equations, J. Phys. A 50 (2017) 204002 [arXiv: 1608.06504] [INSPIRE].

[4] Y. Jiang and Y. Zhang, Algebraic geometry and Bethe ansatz. Part I. The quotient ring for BAE, JHEP 03 (2018) 087 [arXiv:1710.04693] [INSPIRE].

[5] J.L. Jacobsen, Y. Jiang and Y. Zhang, Torus partition function of the six-vertex model from algebraic geometry, JHEP 03 (2019) 152 [arXiv: 1812.00447] [INSPIRE].

\footnotetext{
${ }^{13}$ We have checked this explicitly for small values of $N$, and we expect that it can be proved by induction in $N$, similarly to the case of the closed chain [13].
} 
[6] Z. Bajnok, J.L. Jacobsen, Y. Jiang, R.I. Nepomechie and Y. Zhang, Cylinder partition function of the 6-vertex model from algebraic geometry, arXiv:2002.09019 [INSPIRE].

[7] Z. Bajnok, J.L. Jacobsen, Y. Jiang, R.I. Nepomechie and Y. Zhang, work in progress.

[8] E. Granet and J.L. Jacobsen, On zero-remainder conditions in the Bethe ansatz, JHEP 03 (2020) 178 [arXiv:1910.07797] [INSPIRE].

[9] V. Pasquier and H. Saleur, Common Structures Between Finite Systems and Conformal Field Theories Through Quantum Groups, Nucl. Phys. B 330 (1990) 523 [inSPIRE].

[10] L.D. Faddeev, How algebraic Bethe ansatz works for integrable model, in Relativistic gravitation and gravitational radiation. Proceedings, School of Physics, Les Houches, France, 26 September-6 October 1995, pp. 149-219 (1996) [hep-th/9605187] [INSPIRE].

[11] A.G. Izergin and V.E. Korepin, Pauli principle for one-dimensional bosons and the algebraic Bethe ansatz, Lett. Math. Phys. 6 (1982) 283 [InSPIRE].

[12] L.V. Avdeev and A.A. Vladimirov, On exceptional solutions of the Bethe ansatz equations, Theor. Math. Phys. 69 (1987) 1071 [INSPIRE].

[13] R.I. Nepomechie and C. Wang, Algebraic Bethe ansatz for singular solutions, J. Phys. A 46 (2013) 325002 [arXiv: 1304.7978] [INSPIRE].

[14] G.P. Pronko and Y.G. Stroganov, Bethe equations 'on the wrong side of equator', J. Phys. A 32 (1999) 2333 [hep-th/9808153] [INSPIRE].

[15] E. Mukhin, V. Tarasov and A. Varchenko, Bethe algebra of homogeneous XXX Heisenberg model has simple spectrum, Commun. Math. Phys. 288 (2009) 1 [arXiv:0706.0688].

[16] V. Tarasov, Completeness of the Bethe ansatz for the periodic isotropic Heisenberg model, Rev. Math. Phys. 30 (2018) 1840018.

[17] R.I. Nepomechie and C. Wang, Twisting singular solutions of Bethe's equations, J. Phys. A 47 (2014) 505004 [arXiv:1409.7382] [InSPIRE].

[18] J. Thomae, Beiträge zur Theorie der durch die Heinesche Reihe: ... darstellbaren Functionen, J. Reine Angew. Math. 70 (1869) 258.

[19] F.H. Jackson, The basic gamma-function and the elliptic functions, Proc. Roy. Soc. Lond. A 76 (1905) 127.

[20] R.J. Baxter, Eight vertex model in lattice statistics and one-dimensional anisotropic Heisenberg chain. 1. Some fundamental eigenvectors, Annals Phys. 76 (1973) 1 [INSPIRE].

[21] K. Fabricius and B.M. McCoy, Bethe's equation is incomplete for the XXZ model at roots of unity, J. Statist. Phys. 103 (2001) 647 [cond-mat/0009279] [INSPIRE].

[22] R.J. Baxter, Completeness of the Bethe ansatz for the six and eight vertex models, J. Statist. Phys. 108 (2002) 1 [cond-mat/0111188] [INSPIRE].

[23] V.O. Tarasov, On Bethe vectors for the XXZ model at roots of unity, J. Math. Sci. 125 (2005) 242 [math/0306032].

[24] A.M. Gainutdinov, W. Hao, R.I. Nepomechie and A.J. Sommese, Counting solutions of the Bethe equations of the quantum group invariant open XXZ chain at roots of unity, J. Phys. A 48 (2015) 494003 [arXiv: 1505. 02104] [INSPIRE].

[25] E.K. Sklyanin, Boundary Conditions for Integrable Quantum Systems, J. Phys. A 21 (1988) 2375 [INSPIRE].

[26] P. Fendley and H. Saleur, Deriving boundary S matrices, Nucl. Phys. B 428 (1994) 681 [hep-th/9402045] [INSPIRE]. 